\title{
NEW RECORDS OF THE GENUS SNELLENIUS WESTWOOD, 1882 (HYMENOPTERA: BRACONIDAE: MICROGASTRINAE) FROM VIETNAM, WITH DESCRIPTION OF TWO NEW SPECIES
}

\author{
Khuat Dang Long ${ }^{1^{*}}$, Kees van Achterberg ${ }^{2}$ \\ ${ }^{1}$ Institute of Ecology and Biological Resources (IEBR), VAST, *khuatdanglong@iebr.ac.vn \\ ${ }^{2}$ Department of Terrestrial Zoology (RMNH), Naturalis Biodiversity Center, Leiden, Netherlands
}

\begin{abstract}
The small genus Snellenius Westwood, 1882 is newly recorded for Vietnam and two new species are described and illustrated, viz. Snellenius nigellus Long \& van Achterberg sp. n. and S. similis Long \& van Achterberg sp. n.; one new status is Snellenius theretrae (Watanabe, 1937) stat. n.; two newly recorded species for Vietnam's fauna are Snellenius philippinensis (Ashmead, 1904) and S. maculipennis (Szépligeti, 1900). The checklist and distribution of seventeen extant species and one fossil species, Snellenius succinalis Brues, 1933, are given, of those four species are described from the Neotropical region and the remaining species are described from Oriental region. A key to the species of the genus Snellenius from Vietnam is provided.
\end{abstract}

Keywords: Hymenoptera, Braconidae, Microgastrinae, Snellenius, new record, new species, Vietnam.

\section{INTRODUCTION}

Snellenius Westwood, 1882, is a comparatively small genus in the subfamily Microgastrinae (Braconidae). The genus comprises 17 extant species and occurs in the Australasian (3 species), the East Palaearctic (3 species), the Neotropical (4 species) and the Oriental (7 species) regions [9]. As far as known all species of Microgastrinae are endoparasitoids of lepidopteran larvae and the same as most species of the genus Microplitis, species of the genus Snellenius probably could be found from noctuid larvae. The checklist and distribution of seventeen species of the genus Snellenius are given, a compilated key to four species from Vietnam is provided.

\section{MATERIALS AND METHODS}

Little is known of the Braconidae from Vietnam, for several years the first author has been collecting Braconidae from all over Vietnam to get a first understanding of the Braconidae fauna of Vietnam. The specimens of Snellenius were mainly collected by using sweep nets or malaise traps set in more or less open secondary habitats as impoverished forests, gardens and agricultural lands.

For identification of the subfamilies of Braconidae, see van Achterberg (1993) [1]; for diagnosis of Snellenius see Mason (1981) [7], Nixon (1965) [8] and Austin \& Dangerfield (1993) [3]; for separating the similar genera Microplitis Foerster and Snellenius Westwood and for a key to the genera of the family Microgastrinae, see Austin \& Dangerfield (1992) [2]. For the terminology used in this paper, see van Achterberg (1993). All the photographs were made by the first author with a Canon G15 digital camera attached to an Olympus SZ61 binocular microscope; the measurements of wasps were made under an Olympus SZ40 binocular microscope. Type specimens (holotypes and paratypes) are kept in the Vietnam National Museum of Nature (VNMN), Ha Noi, Vietnam or in Naturalis Biodiversity Center, Leiden (RMNH); VAST stands for Vietnam Academy of Science and Technology.

\section{RESULTS AND DISCUSSION}

\section{Systematics}

Snellenius Westwood, 1882 (Figs 1-22)

Snellenius Westwood, 1882: 19. Typespecies (by original designation): Snellenius vollenhovii Westwood, 1882.

\section{Diagnosis}

Snellenius has prepectal carina complete 
and distinctly developed (figs 4, 8, 13, 19) (absent in closely related genus Microplitis Foerster, 1862); notauli deeply depressed and crenulate or coarsely sculptured (figs 3, 9, 14, 20) (but less developed in Snellenius maculipennis and $S$. clavitergum and in Microplitis); fifth-eighth antennal segments more or less enlarged, occasionally strongly compressed in males. Clypeal margin straight or slightly concave; eye large and densely setose; laterally pronotum with broad and coarsely crenulate oblique furrow; mesoscutum shiny, punctate-rugulose, coriaceous or nearly smooth; scutellar sulcus deep and broad with 1-4 carinae; scutellum coarsely rugose and laterally carinate; propodeum with complete mediolongitudinal carina and surface rugose or coarsely areolate/reticulate-rugose. As in Microplitis, Snellenius has small hind coxa, not reaching to apex of first metasomal tergite (fig. 1); hind tibia widened medially, hind tibial spurs subequal in length; tarsal claws simple. Fore wing areolet present, areolet comparatively large, triangular or quadrangular; fore wing vein 1-R1 short, 1-CU1 much shorter than 2-CU1 (figs 11, 16, 22); hind wing with vanal lobe convex and setose. Metasoma short and stout, first metasomal tergite much longer than wide, with longitudinal trough and superficially sculptured; second-third tergite smooth, hardly separated from one another (fig. 4); hypopygium sparsely setose, evenly sclerotised; ovipositor short (figs 10, 15, 21).

\section{Checklist and distribution}

Snellenius atratus Shenefelt, 1968, from Peru;

Snellenius basalis (Walker, 1874) from Japan; Peru;

Snellenius bicolor Shenefelt, 1968, from

\section{Taxonomy}

\section{Key to four species of the genus Snellenius Westwood from Vietnam}

1. Middle lobe of mesoscutum not depressed laterally; body entirely black or blackish brown; POL 1.1 times as long as OOL; scutellar sulcus with indistinct transverse carina anteriorly, mesoscutum with extensive rugosities (fig. 3); mesopleuron smooth medially, rugose-punctate
Snellenius clavitergum Austin \& Dangerfield, 1993, from Papua New Guinea;

Snellenius gelleus Nixon, 1965, from China

Snellenius guizhouensis Luo \& You, 2005, from China;

Snellenius hippotionus Austin \& Dangerfield, 1993, from Papua New Guinea;

Snellenius latigenus Luo \& You, 2005, from

Snellenius maculipennis (Szépligeti, 1900), from India, Queensland, Thailand and Vietnam (S. radicalis (Wilkinson, 1929), from China, seems to be an invalid species, its orange-brown mesosoma (except propodeum; =form $\mathrm{C}$ of Austin \& Dangerfield, 1993) seems not enough to retain it as separate species);

Snellenius nigellus sp. n., from Vietnam;

Snellenius peruensis Shenefelt, 1968, from Peru;

Snellenius philippinensis (Ashmead, 1904), from Indonesia, Malaysia, Philippines and Vietnam;

Snellenius sedlaceki Austin \& Dangerfield, 1993, from Papua New Guinea;

Snellenius similis sp. n., from Vietnam;

Snellenius succinalis Brues, 1933 (Oligocene);

Snellenius theretrae (Watanabe, 1937) stat. synonym of $S$. basalis (Walker) (van Achterberg \& Desmier de Chenon, 2009) but wing colour and finer sculpture (as pointed out by Nixon (1965)) seems to be sufficiently different to retain as valid sp.;

Snellenius tricolor Shenefelt, 1968, from Argentina;

Snellenius vollenhovii Westwood, 1882, from Papua New Guinea. n., from Japan (formerly considered to be 
anteriorly and posteriorly (fig. 4); vein $\mathrm{M}+\mathrm{CU}$ of hind wing distinctly shorter than vein $1-\mathrm{M}$... S. nigellus sp. $\mathrm{n}$.

- Middle lobe of mesoscutum more or less depressed laterally; body bicoloured: brown with mesoscutum yellow or yellowish brown; POL less than OOL or subequal; scutellar sulcus with transverse anterior carina (figs 9, 14, 20); mesopleuron largely smooth medially, finely and sparsely punctate anteriorly and posteriorly (figs 8, 13, 19); vein M+CU of hind wing slightly shotger or as long as (in similes sp. n.) vein 1-M.

2. Notauli wide and deep posteriorly, with medio-longitudinal carina (fig. 20); scutellar sulcus wide, oval, deep and smooth with one median carina; precoxal sulcus wide and largely crenulate; wing venation from vein $\mathrm{m}$-cu to apex dark brown (fig. 22)

S. philippinensis (Ashmead, 1904)

- Notauli wide and flat posteriorly, without medio-longitudinal carina (figs 9, 14); scutellar sulcus narrower with 3 median carinae (figs 9, 14); precoxal sulcus narrow and sparsely crenulate (figs 8,13 ); wing venation from vein 2-SR to apex dark brown (figs 11,16 )

3. Middle lobe of mesoscutum distinctly depressed laterally (fig. 9); mesopleuron sparsely and finely punctate anteriorly; parastigma 1.0-1.1 times as long as vein 1-SR; pterostigma 2.3 times as long as wide, and its width 1.6-1.7 times vein $\mathrm{r}$ (fig. 11) S. similis sp. $\mathrm{n}$.

- Middle lobe of mesoscutum weakly depressed laterally (fig. 14); mesopleuron densely punctate anteriorly; parastigma 1.3-1.5 times as long as vein 1-SR; pterostigma 2.6-2.7 times as long as wide, and its width 1.3 times vein $\mathrm{r}$ (fig. 16)...

.S. maculipennis (Szépligeti, 1900)

\section{Snellenius nigellus sp. n. (Figs 1-6)}

Material: Holotype, male (VNMN), Mic.1247, NW Vietnam: Hoa Binh, Mai Chau, Tan Son, garden, 2043'103N 104'59'470E, 650 m, MT 1-5.vii.2010, K.D.Long.

Description: Holotype, male, body length $3.0 \mathrm{~mm}$, fore wing length $3.2 \mathrm{~mm}$, antenna 4.1 $\mathrm{mm}$.

Head: Face densely punctate medially, rugose laterally; clypeus finely punctate; temple rugose-punctate; in lateral view width of temple as long as width of eye, in dorsal view height of eye 1.4 times as long as temple (10:7).

Third antennal segment as long as fourth; seventh antennal segments 1.1 times longer than wide (10:9); penultimate antennal segment as long as apical segment; width of face 0.72 length of face and clypeus combined (18:25); clypeus slightly concave; malar space 1.5 times as long as mandible width (6:4); distance between tentorial pits 3.3 times distance between pits and eyes (10:3); in dorsal view, height of eyes 1.25 times as high as temple $(10: 8)$; in lateral view width of eye 0.7 times as long as temple (7:10); ocelli in rather low triangle, anterior ocellus touching anterior tangent of posterior ocelli, POL 1.2 times as long as OOL; POL:Od:OOL=7:2:6; distance between front and hind ocelli 0.46 times as long as OOL (fig. 2).

Mesosoma: Length of mesosoma 1.1 times as long as high (62:58); pronotal trough shiny, smooth; mesopleuron smooth medially, strongly punctate anteriorly and posteriorly (fig. 4); precoxal sulcus wide, deep, sparsely crenulate (fig. 4); medial lobe of mesonotum rugose with medial crenulated groove; notauli deep, sparsely crenulate (fig. 3); scutellar sulcus with 3 carinae, weakly carinate anteriorly, 0.4 times as long as scutellum; scutellum strongly rugose; propodeum with medial carina and lateral transverse rugosities, gradually lowered medially.

Wings: Fore wing: pterostigma 2.6 times as long as wide; vein $r$ of fore wing arising from middle of pterostigma; vein $\mathrm{r} 0.75$ times as long as vein 2-SR and 2.0 times vein 3-SR (r:2-SR:3$\mathrm{SR}=6: 8: 3) ; \quad 1-\mathrm{CU1}: \mathrm{cu}-\mathrm{a}: 2-\mathrm{CU} 1=3: 5: 10$; fore vein $2-\mathrm{SR}+\mathrm{M} 0.7$ times vein $\mathrm{r}$ and 0.4 times vein $\mathrm{m}-\mathrm{cu}$ (4:6:9). Hind wing: vein $\mathrm{M}+\mathrm{CU}$ distinctly shorter vein 1-M (0.6 times); M+CU:1-M:1r-m=14:22:7.

Legs: Length of hind femur: tibia: 
basitarsus: tarsus $=35: 42: 20: 41$; length of hind femur, tibia and basitarsus 3.5, 5.1 and 4.5 times as long as their width, respectively; inner hind tibial spur 0.4 times as long as basitarsus (8:20).

Metasoma: First tergite slightly and roundly narrow apically, granule-rugose, 2.6 times as long as apical width; (fig. 6); second suture weak; second and third metasomal tergites shiny smooth; cerci long, sparsely setose ventrally (fig. 5).

Colour: Blackish brown, fore leg reddish yellow, except taxa brown; middle leg dark brown; hind leg black; first tergite black, second and third tergites white, except medial area dark brown; fore wing vein brown, around vein $r$ beneath stigma smoky brown; second submarginal cell transparent brown.

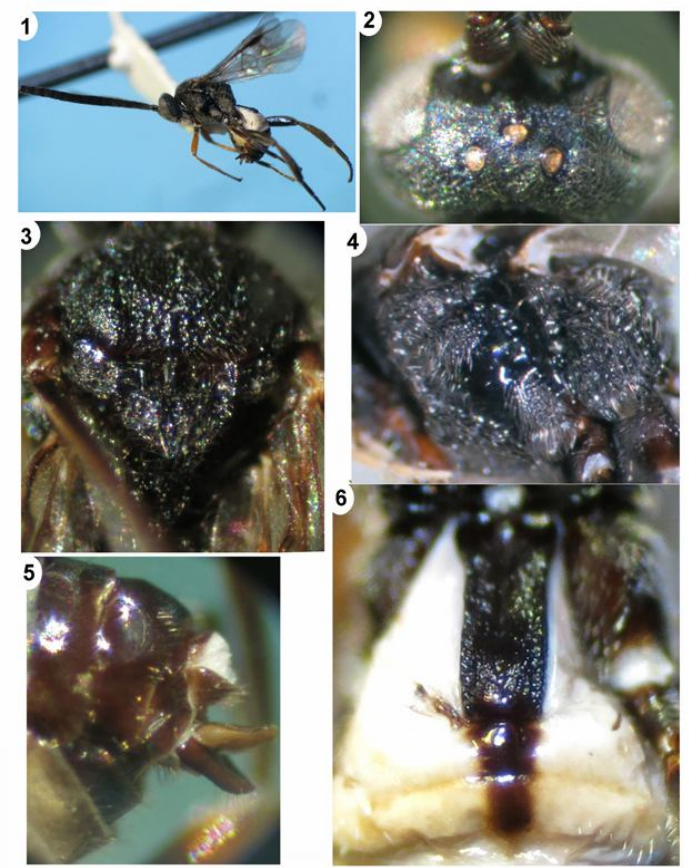

Figures 1-6. Snellenius nigellus Long \& van Achterberg sp. $\mathrm{n}$.

1. Male (habitus); 2. Head (dorsal view);

3. Mesonotum; 4. Mesopleuron; 5. Apex of metasoma (lateral view); 6. Metasomal tergites $1+2+3$.

Female: Unknown.

Etymology: From "niger" (Latin for black, dark, dusky), because of dark colour of body.

Host: Unknown.
Notes: The new species differs from other species by having its body entirely dark brown; the scutellar sulcus with three carinae and anteriorly weakly carinate; the mesopleuron shiny and smooth medially, strongly punctate anteriorly and posteriorly and the hind tibia about 5 times as long as wide (males of $S$. similis and $S$. maculipennis have the hind tibia more swollen dorso-subapically and 3.5 and 3.8 times as long as wide, respectively).

Snellenius similis sp. n. (Figs 7-11)

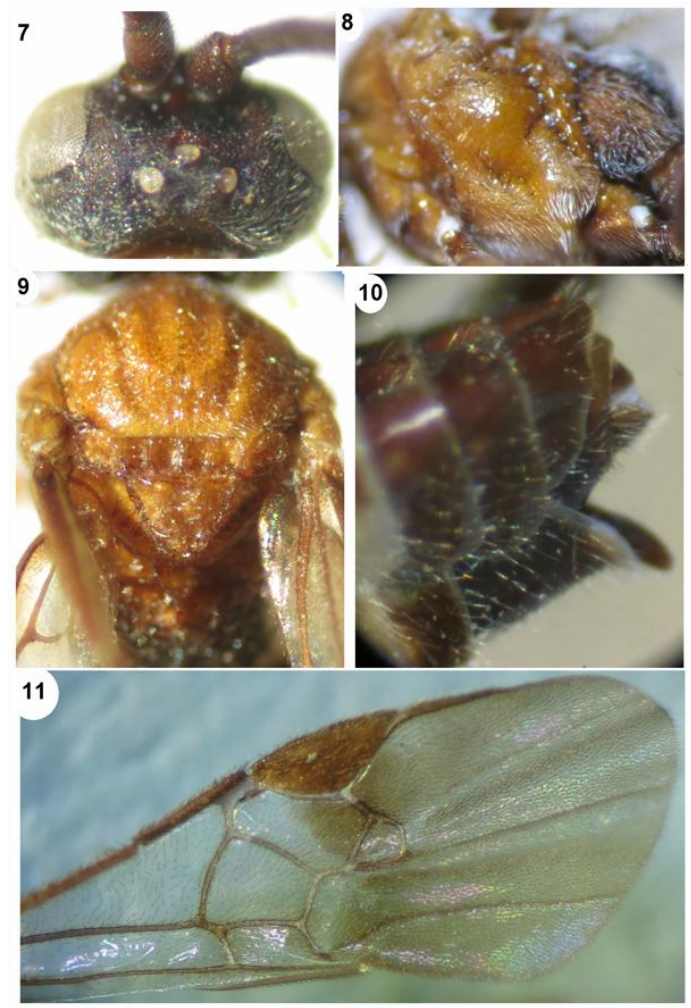

Figures 7-11. Snellenius similis Long \& van Achterberg sp. $n$.

7. Head (dorsal view); 8. Mesopleuron;

9. Mesonotum; 10. Hypopygium; 11. Fore wing.

Material. Holotype, female (VNMN), Mic.1013, N Vietnam: Ha Noi, Gia Lam, Da Ton garden, $20^{\circ} 59^{\prime} 13 \mathrm{~N} 105^{\circ} 55^{\prime} 59 \mathrm{E} 10 \mathrm{~m}$, MT 20-30.v.2001, K.D.Long; Paratypes (VNMN), 2 females, Mic.1012, ibid., but 10-20.iv.2007, K.D.Long; Mic.1437, Vinh Phuc, Tam Dao NP, 1000m 2127’06N 105³8’65E, MT 10-20.vi.2012, K.D.Long; 1 
male, Mic.1368, N Vietnam, Ha Noi, Gia Lam, wild grass and flower, sweep net, 16.iv.2013, D.T. Hoa.

Description: Holotype, female, body length $3.4 \mathrm{~mm}$, fore wing length $3.3 \mathrm{~mm}$, antenna $4.1 \mathrm{~mm}$.

Head: Face rugose-punctate; clypeus finely punctate; frons and vertex rugose; temple setose, rugose; third antennal segment as long as fourth; middle segments 1.25 times longer than wide (5:4); penultimate antennal segment as long as apical segment; width of face 0.8 times length of face and clypeus combined (19:25); malar space 2.0 times as long as mandible width (8:4); distance between tentorial pits 5.0 times distance between pits and eyes (10:2); in lateral view width of temple 1.2 as long as width of eye (11:9); in dorsal view, height of eyes 1.1 times as high as temple (11:10); ocelli in rather high triangle, anterior ocellus not touching anterior tangent of posterior ocelli; POL as long as OOL; POL:Od:OOL=7:3:7 (fig. 7); distance between front and hind ocelli 0.46 times as long as OOL.

Mesosoma: Length of mesosoma 1.3 times as long as high (65:50); pronotal trough rugose; mesopleuron largely smooth medially, finely rugose anteriorly and posteriorly; precoxal sulcus deep, sparsely crenulate (fig. 8); notauli deep, crenulate anteriorly, flat and rugose posteriorly, united posteriorly forming short medial carina near scutellar sulcus (fig. 9); medial lobe of mesoscutum depressed laterally, with medial crenulate groove; scutellar sulcus with 3 carinae, 0.46 times as long as scutellum (6:13); scutellum areolate; propodeum areolaterugose and gradually lowered medially.

Wings: Fore wing: pterostigma 2.3 times as long as wide (25:11); vein $r$ of fore wing arising from middle of pterostigma; vein $r$ of fore wing 0.7 times as long as vein 2-SR and 1.4 times vein $3-\mathrm{SR}$, vein $\mathrm{r}-\mathrm{m}$ as long as vein $3-\mathrm{SR}$ (fig. 11); 1-CU1:cu-a:2-CU1=3:6:12; vein 2-SR+M 0.8 times vein $r$ and 0.55 times as long as vein $\mathrm{m}$-cu; length of first discal cell 1.1 times its height (fig. 11). Hind wing: vein $\mathrm{M}+\mathrm{CU}$ shorter vein $1-\mathrm{M}$ (0.8 times); vein $\mathrm{M}+\mathrm{CU}: 1-\mathrm{M}: 1 \mathrm{r}-\mathrm{m}=$ 20:24:7.
Legs: Length of hind femur:tibia: basitarsus: tarsus $=40: 46: 20: 47$; length of hind femur, tibia and basitarsus 3.6, 4.6 and 4.0 times as long as their width, respectively; inner hind tibial spur 0.4 times as long as basitarsus (8:20).

Metasoma: First tergite 2.3 times apical width (21:13); first metasomal tergite rugose, smooth apically; second and third metasomal tergites shiny smooth, second suture indistinct; hypopygium short, ovipositor sheath sparsely setose apically (fig. 10).

Colour: Brown; antenna brown, scapus yellowish brown; clypeus reddish yellow; mesonotum yellow; propodeum pale brown; fore leg yellow, except trochanter dusty yellow; middle leg brownish yellow, except coxa yellow; hind leg brown; metasoma brown; tergites 1 brown; tergites $2+3$ white, except medial area brown.

Variation: Male: body length 3.4-3.9 mm, fore wing length $3.3-3.5 \mathrm{~mm}$, antenna $3.9-4.3$ mm; middle segments 1.3-1.4 times longer than wide; penultimate antennal segment 0.8-0.9 times as long as apical segment; length of hind femur, tibia and basitarsus 3.0, 3.8 and 4.1 times as long as their width, respectively.

Etymology: Named "similis" (Latin for like, resembling), because the new species is similar to C. maculipennis.

Host: Unknown.

Notes: The new species is close to Snellenius maculipennis (Szépligeti, 1900), from India, Australia (Queensland) and Thailand, but differs from the later by having: mesopleuron sparsely and finely punctate anteriorly (more or less densely punctate anteriorly in S. maculipennis); length of pterostigma 2.3 times as long as its width, and pterostigma width 1.6-1.7 times vein $\mathrm{r}$ (2.6-2.7 times and 1.3 times in $S$. maculipennis, respectively); vein 1-SR of fore wing subequal parastigma (0.75-0.80 times in S. maculipennis).

Snellenius maculipennis (Szépligeti, 1900) (Figs 12-17)

Material: 2 female, (VNMN), Mic.1303, Mic.1304, N Vietnam: Ha Noi, Long Bien, garden, MT 01-10.viii.2012, K.D. Long, 
D.T.Hoa; 5 males (VNMN, RMNH), Mic.1300, ibid. but 01-10.vi.2012; Mic.612, N Vietnam: Ha Noi, Gia Lam, Da Ton garden, 2059'13N $105^{\circ} 55^{\prime} 59 \mathrm{E} \quad 10 \mathrm{~m}, \quad \mathrm{MT}$ 15-25.xi.2001, K.D.Long; Mic.613, ibid. but 25.xi-05.xii.2001; Mic.950, ibid. but 05-15.ix.2001; Mic.951, ibid. but 25.x-05.xi.2001, K.D.Long.

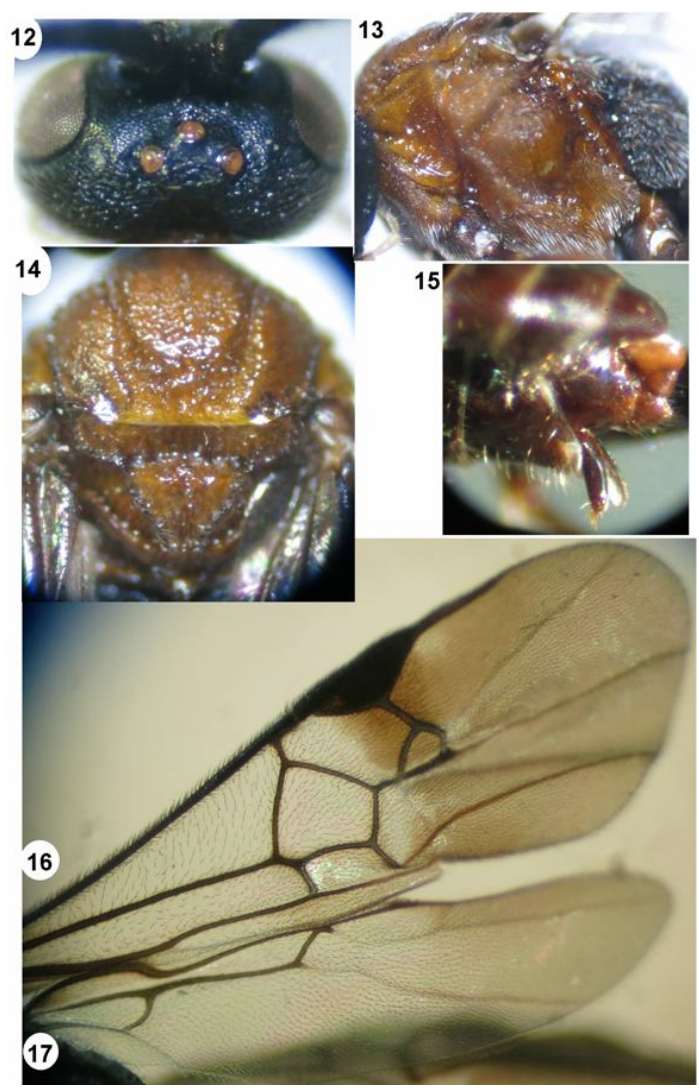

Figures 12-17. Snellenius maculipennis (Szépligeti, 1900)

12. Head (dorsal view); 13. Mesopleuron; 14. Mesonotum; 15. Hypopygium; 16. Fore wing; 17. Hind wing.

Diagnosis: Female, body length $3.4-4.0 \mathrm{~mm}$, fore wing length 3.3-3.7 mm, antenna 4.0-4.2 $\mathrm{mm}$. Male, body length $3.6-4.0 \mathrm{~mm}$, fore wing length $3.7 \mathrm{~mm}$, antenna 4.5-4.7 mm. Clypeus dark brown, scapus brown; ocelli in rather high triangle, anterior ocellus not touching anterior tangent of posterior ocelli, POL 0.85 times as long as OOL (fig. 12); distance between front and hind ocellus 0.43 times OOL; pronotal trough more or less crenulate anteriorly; notauli deep, wide and rugose, united posteriorly not forming short medial carina near scutellar sulcus; medial lobe of mesoscutum more flat laterally (fig. 14); scutellar sulcus with $3^{+}$carinae; precoxal sulcus sparsely crenulate (fig. 13); propodeum gradually lowered medially and with complete median carina. Fore wing: length of pterostigma 2.6 times its width; vein $r$ of fore wing 0.8 times as long as vein $2-S R$ and 2.0 times vein 3-SR, vein r-m shorter vein 3$\mathrm{SR}$ (fig. 16); vein 2-SR+M 0.75 times vein $\mathrm{r}$ and 0.5 as long as vein $\mathrm{m}-\mathrm{cu}$; length of first discal cell as long its height (fig. 16). Hind wing: vein $\mathrm{M}+\mathrm{CU} 0.85$ times $1-\mathrm{M}$ and 3.5 times as long as vein 1r-m (fig. 17). Legs, female: length of hind femur, tibia and basitarsus 3.4, 4.1 and 3.8 times as long as their width, respectively; male: length of hind femur, tibia and basitarsus 3.1, 3.5 and 3.9 times as long as their width, respectively. Hypopygium short, ovipositor sheath sparsely setose apically (fig. 15).

Host: Unknown.

Snellenius philippinensis (Ashmead, 1904) (Figs 18-22)

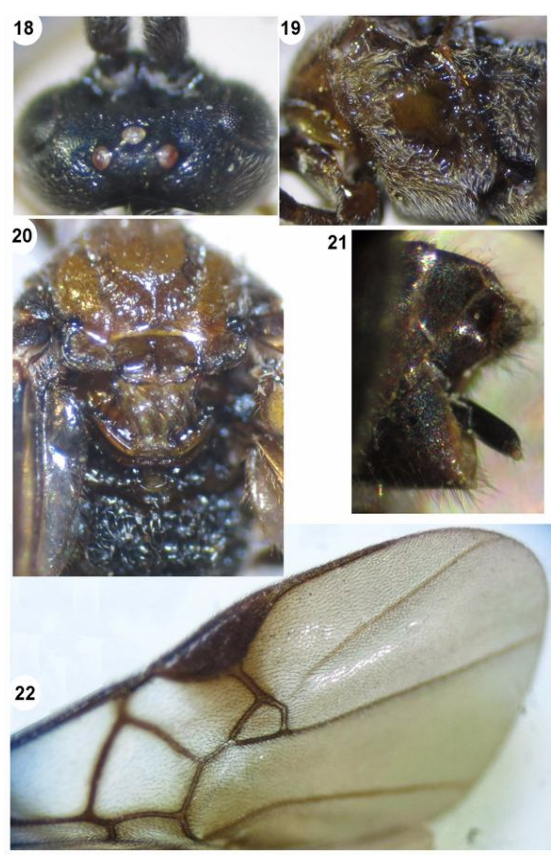

Figures 18-22. Snellenius philippinensis (Ashmead, 1904)

18. Head (dorsal view), 19. Mesopleuron, 20. Mesonotum and propodeum, 21. Hypopygium, 22. Fore wing. 
Material: 15 females (VNMN), Mic.291, S Vietnam: Lam Dong, Duc Trong, Hiep An, $11^{\circ} 50^{\prime} 34 \mathrm{~N} 108^{\circ} 27^{\prime} 25 \mathrm{E} 1095 \mathrm{~m}$, swept from black bean, K.D.Long; Mic.1124, N Vietnam: Ha Noi, Gia Lam, Da Ton, garden, 2059' $13 \mathrm{~N}$ 105'55'59E 10 m, MT 04-14.vi.2001, K.D. Long; Mic.1219-1225, NW Vietnam: Hoa Binh, Mai Chau, Tan Son, garden $20^{\circ} 43^{\prime} 103 \mathrm{~N}$ 10459'470E $650 \mathrm{~m}$, MT 10-15.viii.2010, K.D.Long; Mic. 1235, ibid. but 10-15.v.2010; Mic.1236, ibid. but 01-05.vii.2010; Mic.1238, ibid. but 10-15.viii.2010; Mic.1239, ibid. but 01-05.vii. 2010; Mic.1254-1256, ibid. but 2025.vii.2010; 2 females (RMNH), N Vietnam: Ninh Binh, Cuc Phuong NP, Malaise trap near centre, c. $225 \mathrm{~m}$, 14.iv-1.v.2000 and 1527.v.2000, Mai Phu Quy.

Diagnosis: Female. Body length 4.8-5.8 mm, fore wing length 4.6-5.5 mm, antenna 5.0-5.8 $\mathrm{mm}$. Clypeus reddish yellow, scapus yellow ventrally. Ocelli in rather low triangle, anterior ocellus touching anterior tangent of posterior ocelli, POL 0.9 times as long as OOL; distance between front and hind ocellus 0.43 as long as OOL (fig. 18); medial lobe of mesoscutum distinctly elevated above lateral lobes, notauli broad and deep, united posteriorly forming medio-longitudinal carina (fig. 20); scutellar sulcus largely oval-shaped and with one carina; mesopleuron smooth medially; precoxal sulcus sparsely carinate (fig. 19); dorsal face of propodeum distinctly angled with posterior face. Fore wing: length of pterostigma 2.5-2.6 times its width; vein $r$ of fore wing 0.75 times as long as vein $2-\mathrm{SR}$ and 1.8 times vein $3-\mathrm{SR}$, vein $\mathrm{r}-\mathrm{m}$ longer vein 3-SR (fig. 22); vein $2-\mathrm{SR}+\mathrm{M}$ as long as vein $\mathrm{r}$ and 0.64 times as long as vein $\mathrm{m}-\mathrm{cu}$ (fig. 22); length of first discal cell subequal its height. Hind wing: vein $\mathrm{M}+\mathrm{CU} 0.8$ times $1-\mathrm{M}$ and 2.2 times as long as vein 1r-m. Legs, female: length of hind femur, tibia and basitarsus 3.5, 4.8 and 4.4 times as long as their width, respectively. Hypopygium short, ovipositor sheath sparsely setose apically (fig. 21).

Male: Unknown.

Host: Unknown.

Acknowledgements: This research is funded by Vietnam Foundation for Science and
Technology Development (NAFOSTED) under grant number 106.15-2012.62. We express sincere thanks to Dr. Mai Phu Quy and MSc Dang Thi Hoa (IEBR) for providing a part of material.

\section{REFERENCES}

1. Achterberg van C., 1993. Illustrated key to the subfamilies of the Braconidae (Hymenoptera: Ichneumonoidea). Zoologische Verhandelingen Leiden, 283: 1-189.

2. Austin A. D., Dangerfield P. C., 1992. Synopsis of Australasian Microgastrinae (Hymenoptera: Braconidae), with a key to genera and description of new taxa. Invertebrate Taxonomy, 6(1): 1-76.

3. Austin A. D., Dangerfield P. C., 1993. Systematics of Australian and New Guinean Microplitis Foester and Snellenius Westwood (Hymenoptera: Braconidae: Microgastrinae), with a Review of their Biology and Host Relationship. Invertebrate Taxonomy, 7: 1097-1166.

4. Khuat Dang Long, 2007. Three new species of the subfamily Microgastrinae (Hymenoptera: Braconidae) from Viet Nam. TAP CHI SINH HOC, 29(3): 25-31.

5. Khuat Dang Long, Belokobylskij S. A., 2003. A preliminary list of the Braconidae (Hymenoptera) of Vietnam. Russian Entomological Journal, 12(4): 385-398.

6. Luo Qing-Huai, You Lan-Shao, 2005. Descriptions of two new species of the genus Slenellenius Westwood (Hymenoptera, Braconidae, Microgastrinae) from China. Acta Zootaxonomica Sinica, 30(1): 170-174.

7. Mason W. R. M., 1981. The polyphyletic nature of Apanteles Foerster (Hymenoptera: Braconidae): A phylogeny and reclassification of Microgastrinae. Mem. Entom. Soc. Canada, 115: 1-147.

8. Nixon G. E. J., 1965. A reclassification of the tribe Microgasterini (Hymenoptera: Braconidae). Bull. Br. Mus. nat. Hist. Entomology, Suppl. 2, 1-284. 
9. Yu D. S., Achterberg van K., Horstmann K., 2013. Ichneumonoidea 2012 (Biological and taxonomical information), Taxapad Interactive Catalogue, Ottawa.
10. Westwood J. O., 1882. Descriptions of new or imperfectly known species of Ichneumones Adsciti. Tijdschrift voor Entomologie 25: 17-48.

\title{
GHI NHẬN MỚI GIỐNG SNELLENIUS WESTWOOD, 1882 \\ (HYMENOPTERA: BRACONIDAE: MICROGASTRINAE) Ở VIẸTT NAM VÀ MÔ TẢ HAI LOÀI MỚI CHO KHOA HỌC
}

\author{
Khuất Đăng Long ${ }^{1}$, Kees van Achterberg ${ }^{2}$ \\ ${ }^{1}$ Viện Sinh thái và Tài nguyên sinh vật, Viện Hàn lâm KH \& CN Việt Nam \\ ${ }^{2}$ Department of Terrestrial Zoology (RMNH), Naturalis Biodiversity Center, Leiden, Netherlands
}

\section{TÓM TẮT}

Giống Snellenius Westwood, 1882 gồm các loài ong ký sinh thuộc phân họ ong bụng nhỏ Microgastrinae, họ ong ký sinh Braconidae. Đây là một giống hiếm gặp, đển nay trên thế giới mới thống kê được 17 loài hiện còn và một loài hóa thạch Snellenius succinalis Brues, 1933, trong số đó có 4 loài phân bố ở Nam Mỹ, số loài còn lại được mô tả từ khu hệ vùng Đông Phương. Có 2 loài mới cho khoa học được mô tả từ khu hệ của Việt Nam: Snellenius nigellus Long \& van Achterberg sp. n. and S. similis Long \& van Achterberg sp. n.; ghi nhận mới hai loài từ khu hệ của Việt Nam: Snellenius philippinensis (Ashmead, 1904) and S. maculipennis (Szépligeti, 1900); 1 loài được xếp lại, Snellenius theretrae (Watanabe, 1937) stat. n..

Trong phân họ Microgatrinae, hai giống Snellenius Westwood và Microplitis Foerster, 1862 có một số đặc điểm hình thái rất giống nhau như đốt háng sau nhỏ, đỉnh đốt này không dài vượt quá tấm lưng bụng 1 (hình 1); cánh trước có ô cánh submarginal 2 nhỏ (hình 11, 16, 22); máng đẻ trứng ngắn; bao máng đẻ trứng có lông măng thưa ở đỉnh (hình 10,15,21). Giống Snellenius có thể được phân biệt do phía trước sườn bên ngực giữa (mesopleuron) có gờ viền nổi rõ (prepectal carina) (hình 4, 8, 13, 19); rãnh lưng lõm rõ, nhăn hoặc có gờ; rãnh lõm trước scutellum (scutellar sulcus) rộng và sâu, có từ 1-4 gờ dọc (hình $3,9,14,20$ ). Những đặc điểm khác biệt về hình thái của 4 loài thuộc giống Snellenius ở Việt Nam có thể tìm thấy trong khóa định loại.

\section{Đặc điểm chẩn loại}

Snellenius nigellus Long \& van Achterberg sp. n. (Hình 1-6)

Con đực: Toàn bộ cơ thể đen hoặc nâu đen. Ba mắt đơn ở vị trí thấp, rìa sau mắt đơn trước cắt tiếp tuyến rìa trước của hai mắt đơn sau (hình 2). Sườn ngực giữa có phần nhẵn ở trung tâm, phần trước và sau có chấm lỗ thô và dày; rãnh lưng sâu và có khía nhăn; rãnh lõm phía trước scutellum có 3 gờ dọc (hình 3 ). Cánh trước có chiều dài mắt cánh bằng 2,6 lần chiều rộng, gân $\mathrm{r}$ bằng 0,75 lần chiều dài gân 2-SR và 2,0 lần gân 3-SR; cánh sau có gân $\mathrm{M}+\mathrm{CU}$ ngắn hơn rõ gân $1-\mathrm{M}$ (bằng 0,6 lần). Chiều dài đốt đùi, ống chân và bàn 1 chân sau tương ứng dài hơn chỗ rộng nhất của các đốt này là 3,$5 ; 5,1$ và 4,5 lần; ống chân sau hơi phồng ở gần sát đỉnh. Tấm lưng bụng 1 nhăn, hơi hẹp sát đỉnh (hình 6).

\section{Snellenius similis Long \& van Achterberg sp. n. (Hình 7-11)}

Con cái: Cơ thể màu nâu trừ đốt ngực trước màu vàng. Ngực giữa màu vàng; ba mắt đơn ở vị trí khá cao, rìa sau mắt đơn trước cách xa tiếp tuyến rìa trước của hai mắt đơn sau (hình 7). Sườn ngực giữa có phần nhẵn rộng ở trung tâm, phần phía trước và sau có chấm lỗ mịn và thưa (hình 8 ); rãnh lưng sâu, có khía ở phía trước, phía sau rộng hơn và nhăn; rãnh lõm phía trước scutellum có 3 gờ dọc (hình 9). Cánh trước có mắt cánh rộng, ô cánh submarrginal 2 khá lớn (hình 11 ); chiều dài mắt cánh bằng 2,3 lần chiều rộng còn chiều rộng mắt cánh bằng 1,6-1,7 lần chiều dài gân $\mathrm{r}$; gân $\mathrm{r}$ bằng 0,7 lần gân 2-SR và 1,4 lần chiều dài gân 3 -SR; cánh sau có gân 
$\mathrm{M}+\mathrm{CU}$ hơi ngắn hơn gân 1-M (bằng 0,8 lần). Chiều dài đốt đùi, ống chân và bàn 1 chân sau tương ứng dài hơn chỗ rộng nhất của các đốt này là 3,6; 4,6 và 4,0 lần. Con đực: thân dài 3,4-3,9 mm; cánh trước dài 3,3-3,5 $\mathrm{mm}$; râu dài $3,9-4,3 \mathrm{~mm}$; chiều dài đốt đùi, ống chân và bàn 1 chân sau tương ứng dài hơn chỗ rộng nhất của các đốt này là 3,$0 ; 3,8$ và 4,1 lần. Mấu ôm bao máng đẻ trứng ngắn có phủ lông măng khá dày và cứng; bao máng đẻ trứng ngắn, có lông măng thưa ở đỉnh (hình 10).

\section{Snellenius maculipennis (Szépligeti, 1900) (Hình 12-17)}

Con cái: Cơ thể màu nâu trừ đốt ngực trước màu vàng. Ngực giữa màu vàng; ba mắt đơn ở vị trí khá cao, rìa sau mắt đơn trước cách xa tiếp tuyến rìa trước của hai mắt đơn sau (hình 12 ). Sườn ngực giữa có phần nhẵn khá rộng ở trung tâm, phần phía trước và sau có chấm lỗ mịn và thưa (hình 13 ); rãnh lưng sâu, có khía ở phía trước, phía sau rộng hơn và nhăn; rãnh lõm phía trước scutellum có 3 gờ dọc (hình 14). Cánh trước có mắt cánh hẹp hơn, ô cánh submarrginal 2 trung bình (hình 16); chiều dài mắt cánh bằng 2,6 lần chiều rộng còn chiều rộng mắt cánh bằng 1,3 lần chiều dài gân $\mathrm{r}$; gân $\mathrm{r}$ bằng 0,8 lần chiều dài gân $2-\mathrm{SR}$ và 2,0 lần gân 3$\mathrm{SR}$; cánh sau có gân $\mathrm{M}+\mathrm{CU}$ hơi ngắn hơn gân $1-\mathrm{M}$ (bằng 0,85 lần). Chiều dài đốt đùi, ống chân và bàn 1 chân sau tương ứng dài hơn chỗ rộng nhất của các đốt này là 3,$4 ; 4,1$ và 3,8 lần; con đực: chiều dài đốt đùi, ống chân và bàn 1 chân sau tương ứng dài hơn chỗ rộng nhất của các đốt này là 3,$1 ; 3,5$ và 3,9 lần. Mấu ôm bao máng đẻ trứng ngắn có phủ lông măng cứng, thưa; bao máng đẻ trứng ngắn, có lông măng thưa rải rác ở đỉnh (hình 15).

\section{Snellenius philippinensis (Ashmead, 1904) (Hình 18-22)}

Con cái: Cơ thể màu nâu trừ đốt ngực trước màu nâu nhạt. Ngực giữa màu vàng; ba mắt đơn ở vị trí thấp, rìa sau mắt đơn trước cắt tiếp tuyến rìa trước của hai mắt đơn sau (hình 18). Sườn ngực giữa có phần nhẵn hẹp ở trung tâm, phần phía trước và sau có chấm lỗ mịn và thưa (hình 19); rãnh lưng sâu, hẹp phía trước, rộng phía sau và đều có khía, phía sau được chia đôi bởi một gờ nổi (hình 20); rãnh lõm phía trước scutellum rộng, hình oval có 1 gờ dọc (hình 20). Cánh trước có ô cánh submarrginal 2 rộng (hình 22); chiều dài mắt cánh bằng 2,5-2,6 lần chiều rộng; gân $\mathrm{r}$ bằng 0,75 lần chiều dài gân 2-SR và bằng 1,8 lần gân 3-SR (hình 22); cánh sau có gân $\mathrm{M}+\mathrm{CU}$ hơi ngắn hơn gân $1-\mathrm{M}$ (bằng 0,8 lần). Chiều dài đốt đùi, ống chân và bàn 1 chân sau tương ứng dài hơn chỗ rộng nhất của các đốt này là 3,5; 4,8 và 4,4 lần. Mấu ôm bao máng đẻ trứng tương đối rõ, có phủ lông măng cứng, khá dày; bao máng đẻ trứng ngắn, có lông măng thưa ở đỉnh (hình 21).

Tù khóa: Hymenoptera, Braconidae, Microgastrinae, Snellenius, ghi nhận mới, loài mới, Việt Nam.

Ngày nhận bài: 3-1-2013 